Article

\title{
Generalized orthopair fuzzy matrices
}

\section{Silambarasan}

Department of Mathematics, Sri Manakula Vinayagar Engineering College, Madagadipet, Puducherry-605 107, India; sksimbuking@gmail.com

Academic Editor: Feng Feng

Received: 6 June 2021; Accepted: 22 August 2021; Published: 4 September 2021.

\begin{abstract}
A q-rung orthopair fuzzy matrix (q-ROFM), an extension of the Pythagorean fuzzy matrix (PFM) and intuitionistic fuzzy matrix (IFM), is very helpful in representing vague information that occurs in real-world circumstances. In this paper we define some algebraic operations, such as max-min, min-max, complement, algebraic sum, algebraic product, scalar multiplication $(n A)$, and exponentiation $\left(A^{n}\right)$. We also investigate the algebraic properties of these operations. Furthermore, we define two operators, namely the necessity and possibility to convert q-ROFMs into an ordinary fuzzy matrix, and discuss some of their basic algebraic properties. Finally, we define a new operation(@) on q-ROFMs and discuss distributive laws in the case where the operations of $\oplus_{q}, \otimes_{q}, \wedge_{q}$ and $\vee_{q}$ are combined each other.
\end{abstract}

Keywords: Intuitionistic fuzzy matrix; Pythagorean fuzzy matrix; q-rung orthopair fuzzy matrix; Algebraic sum; Algebraic product; Scalar multiplication; Exponentiation.

MSC: 03E72; 15B15; 15B99.

\section{Introduction}

$\mathbf{T}$ he concept of an intuitionistic fuzzy matrix (IFM) was introduced by Khan et al. [1] and Im et al. [2] to generalize the concept of Thomason's fuzzy matrix [3]. Each element in an IFM is expressed by an ordered pair $\left\langle\mu_{a_{i j}}, v_{a_{i j}}\right\rangle$ with $\mu_{a_{i j}}, v_{a_{i j}} \in[0,1]$ and $0 \leq \mu_{a_{i j}}+v_{a_{i j}} \leq 1$. Since the IFS was proposed, it has received a lot of attention in many fields, such as pattern recognition, medical diagnosis, and so on. But if the sum of the membership degree and the nonmembership degree is greater than 1, the IFM is no longer applicable. Khan and Pal [4] defined some basic operations and relations of IFMs including maxmin, minmax, complement, algebraic sum, algebraic product etc. and proved equality between IFMs. After the introduction of IFM theory, many researchers attempted the important role in IFM theory [5-14].

Yager [15] introduced the concept of a Pythagorean fuzzy set (PFS) and developed some aggregation operations for PFS. Zhang and Xu [16] studied various binary operations over PFS and also proposed a decision making algorithm based on PFS. Recently, Yager [17] proposed the concept of the q-ROFS, in which MD $u$ and NMD satisfy $\mu^{q}+v^{q} \leq 1(q \geq 1)$. We can see that the IFS and PFS are special cases of q-ROFS. As q-rung increases, the range of processing fuzzy information increases. In recent years, the topic of information aggregation has attracted a lot of attention and is one of the key research issues in the problems of MAGDM. As far as q-ROFS is concerned, different aggregation operators have been introduced and applied, such as q-ROFWA and q-ROFWG operator [18]. After the introduction of q-ROFS theory, many researchers attempted the important role in PFS and q-ROFS theory [19-25].

Using the theory of PFS ans q-ROFS, Silambarasan and Sriram [26] defined the Pythagorean fuzzy matrix (PFM) theory and its algebraic operations. Each element in an PFM is expressed by an ordered pair $\left\langle\mu_{a_{i j}}, v_{a_{i j}}\right\rangle$ with $\mu_{a_{i j}}, v_{a_{i j}} \in[0,1]$ and $0 \leq \mu_{a_{i j}}^{2}+v_{a_{i j}}^{2} \leq 1$. Also,they constructed $n A$ and $A^{n}$ of a Pythagorean fuzzy matrix $A$ and using these operations. Further, they defined the commutative monoid on Pythagorean fuzzy matrices and proved that the set of all PFMs forms a commutative monoid [27]. After the introduction of PFM theory, many researchers worked in PFM and Fermatean fuzzy matrix theory [28]. Since the PFM was brought up, it has been widely applied in FM operations on q-ROFMs and prove their desirable properties. In Section 5, we define necessity and possibility on q-ROFMs and proved some algebraic properties of these operations. In Section 6, we define a new operation(@) on q-ROFMs and investigated their algebraic properties. We write the conclusion of the paper in the last section. 


\section{Preliminaries}

In this section, some basic concepts related to the intuitionistic fuzzy matrix (IFM) and Pythagorean fuzzy matrix (PFM) have been given.

Definition 1. [1] An intuitionistic fuzzy matrix (IFM) is a pair $A=\left[\left\langle\mu_{a_{i j}}, v_{a_{i j}}\right\rangle\right]$ of a non negative real numbers $\mu_{a_{i j}}, v_{a_{i j}} \in[0,1]$ satisfying $0 \leq \mu_{a_{i j}}+v_{a_{i j}} \leq 1$ for all $i, j$.

Definition 2. [26] A Pythagorean fuzzy matrix (PFM) is a pair $A=\left[\left\langle\mu_{a_{i j}}, v_{a_{i j}}\right\rangle\right]$ of non negative real numbers $\mu_{a_{i j}}, v_{a_{i j}} \in[0,1]$ satisfying the condition $0 \leq \mu_{a_{i j}}^{2}+v_{a_{i j}}^{2} \leq 1$, for all $i, j$. Where $\mu_{a_{i j}} \in[0,1]$ is called the degree of membership and $v_{a_{i j}} \in[0,1]$ is called the degree of non-membership.

\section{3. q-rung orthopair fuzzy matrices (q-ROFMs)}

In this section, we briefly introduce the q-rung orthopair fuzzy matrices and give examples.

Definition 3. A q-rung orthopair fuzzy matrix (q-ROFM) is a pair $A=\left[\left\langle\mu_{a_{i j}}, v_{a_{i j}}\right\rangle\right]$ of non negative real numbers $\mu_{a_{i j}}, v_{a_{i j}} \in[0,1]$ satisfying the condition $0 \leq \mu_{a_{i j}}^{q}+v_{a_{i j}}^{q} \leq 1(q \geq 1)$, for all $i, j$. Where $\mu_{a_{i j}} \in[0,1]$ is called the degree of membership and $v_{a_{i j}} \in[0,1]$ is called the degree of non-membership.

For understanding the q-ROFM better, we give an instance to illuminate the understandability of the q-ROFM: We can definitely get $0.9+0.6>1$, and, therefore, it does not follow the condition of intuitionistic fuzzy matrices. Also, we can get $(0.9)^{2}+(0.6)^{2}=0.81+0.36=1.17>1$, which does not obey the constraint condition of Pythagorean fuzzy matrices. However, we can get $(0.9)^{q}+(0.6)^{q} \leq 1(q \geq 1)$, which is good enough to apply the q-ROFM to control it.

Theorem 1. The q-ROFMs is larger than the set of PFMs and IFMs.

Proof. Any intuitionistic fuzzy matrix $\left(\mu_{a_{i j}}, v_{a_{i j}}\right)$ that is an IFM is also a PFM and a q-ROFM. For any two fuzzy matrices $A, B \in[0,1]$, we get $\mu_{a_{i j}}^{q} \leq \mu_{a_{i j}}^{2} \leq \mu_{a_{i j}}$ and $v_{a_{i j}}^{q} \leq v_{a_{i j}}^{2} \leq v_{a_{i j}}$. Thus $\mu_{a_{i j}}+v_{a_{i j}} \leq 1 \Rightarrow \mu_{a_{i j}}^{2}+v_{a_{i j}}^{2} \leq 1 \Rightarrow$ $\mu_{a_{i j}}^{q}+v_{a_{i j}}^{q} \leq 1$. Consider a point $(0.9,0.6)$, we see that $(0.9)^{q}+(0.6)^{q} \leq 1,(q \geq 1)$ thus this is an q-ROFM. Since $(0.9)^{2}+(0.6)^{2}=0.81+0.36=1.17 \geq 1$ and $0.9+0.6 \geq 1$, therefore $(0.9,0.6)$ is neither a PFM nor an IFM.

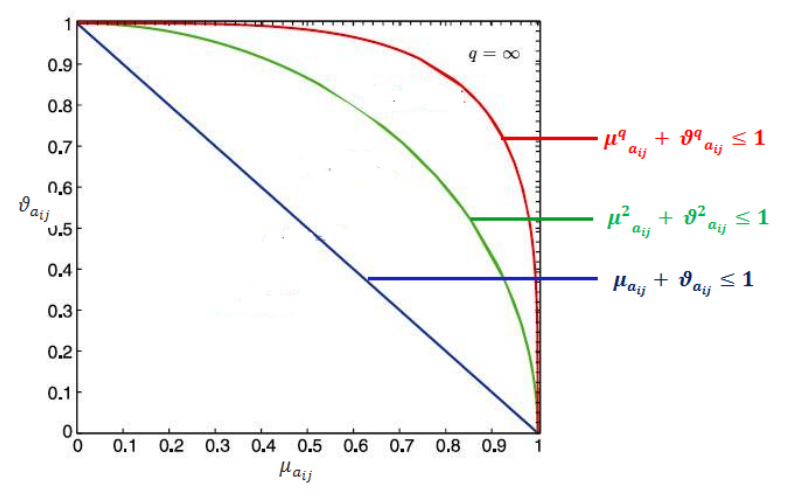

Figure 1. Comarison of grades space of IFM, PFM and q-ROFM

This development can be evidently recognized in Figure 1 . Here we notice that IFMs are all points beneath the line $\mu_{a_{i j}}+v_{a_{i j}} \leq 1$, the PFMs are all points with $\mu_{a_{i j}}^{2}+v_{a_{i j}}^{2} \leq 1$, and the q-ROFMs are all points with $\mu_{a_{i j}}^{q}+v_{a_{i j}}^{q} \leq 1$. We see then that the q-ROFMs enable for the presentation of a bigger body of nonstandard membership function then IFMs and PFMs. Here $Q_{m \times n}$ denote the set of all the q-ROFMs. 


\section{PFM operations on $\mathrm{q}-\mathrm{ROFMs}$}

In this section we propose the definition of q-rung orthopair fuzzy matrix (q-ROFM) and introduce some operations on q-ROFM. Also, we prove some algebraic properties, such as commutativity, associativity, identity, distributivity and De Morgan's laws over complement.

Definition 4. Let $A=\left[\left\langle\mu_{a_{i j}}, v_{a_{i j}}\right\rangle\right]$ and $B=\left[\left\langle\mu_{b_{i j}}, v_{b_{i j}}\right\rangle\right]$ be two q-ROFMs of the same size. Then

(i) $A \vee_{q} B=\left[\left\langle\max \left\{\mu_{a_{i j}}, \mu_{b_{i j}}\right\} \min \left\{v_{a_{i j}}, v_{b_{i j}}\right\}\right\rangle\right]$

(ii) $A \wedge_{q} B=\left[\left\langle\min \left\{\mu_{a_{i j}}, \mu_{b_{i j}}\right\} \max \left\{v_{a_{i j}}, v_{b_{i j}}\right\}\right\rangle\right]$,

(iii) $A^{C}=\left[\left\langle\mu_{a_{i j}}, v_{a_{i j}}\right\rangle\right]$.

Definition 5. Let $A=\left[\left\langle\mu_{a_{i j}}, v_{a_{i j}}\right\rangle\right]$ and $B=\left[\left\langle\mu_{b_{i j}}, v_{b_{i j}}\right\rangle\right]$ be two q-ROFMs of the same size. Then

(i) $A \oplus_{q} B=\left[\left\langle\left(\mu_{a_{i j}}^{q}+\mu_{b_{i j}}^{q}-\mu_{a_{i j}}^{q} \mu_{b_{i j}}^{q}\right)^{1 / q}, v_{a_{i j}} v_{b_{i j}}\right\rangle\right]$,

(ii) $A \otimes_{q} B=\left[\left\langle\mu_{a_{i j}} \mu_{b_{i j}}\left(v_{a_{i j}}^{q}+v_{b_{i j}}^{q}-v_{a_{i j}}^{q} v_{b_{i j}}^{q}\right)^{1 / q}\right\rangle\right]$,

(iii) $n A=\left[\left\langle\left(1-\left(1-\mu_{a_{i j}}^{q}\right)^{n}\right)^{1 / q},\left(v_{a_{i j}}\right)^{n}\right\rangle\right]$,

(iv) $A^{n}=\left[\left\langle\mu_{a_{i j^{\prime}}}^{n}\left(1-\left(1-\mu_{a_{i j}}^{q}\right)^{n}\right)^{1 / q}\right\rangle\right]$,

where,+- and . are ordinary addition, subtraction and multiplication respectively.

Theorem 2. For $A, B \in Q_{m \times n}$, we have

(i) $A \oplus_{q} B=B \oplus_{q} A$,

(ii) $A \otimes_{q} B=B \otimes_{q} A$,

(iii) $n\left(A \oplus_{q} B\right)=n A \oplus_{q} n B, n>0$,

(iv) $\left(n_{1}+n_{2}\right) A=n_{1} A \oplus_{q} n_{2} A, n_{1}, n_{2}>0$,

(v) $\left(A \oplus_{q} B\right)^{n}=A^{n} \otimes_{q} B^{n}, n>0$,

(vi) $A^{n_{1}} \otimes_{q} A^{n_{2}}=A^{\left(n_{1}+n_{2}\right)}, n_{1}, n_{2}>0$.

Proof. (i)

$$
\begin{aligned}
A \oplus_{q} B & =\left[\left\langle\left(\mu_{a_{i j}}^{q}+\mu_{b_{i j}}^{q}-\mu_{a_{i j}}^{q} \mu_{b_{i j}}^{q}\right)^{1 / q}, v_{a_{i j}} v_{b_{i j}}\right\rangle\right] \\
& =\left[\left\langle\left(\mu_{b_{i j}}^{q}+\mu_{a_{i j}}^{q}-\mu_{b_{i j}}^{q} \mu_{a_{i j}}^{q}\right)^{1 / q}, v_{b_{i j}} v_{a_{i j}}\right\rangle\right] \\
& =B \oplus_{q} A .
\end{aligned}
$$

(ii)

$$
\begin{aligned}
A \otimes_{q} B & =\left[\left\langle\mu_{a_{i j}} \mu_{b_{i j}}\left(v_{a_{i j}}^{q}+v_{b_{i j}}^{q}-v_{a_{i j}}^{q} v_{b_{i j}}^{q}\right)^{1 / q}\right\rangle\right] \\
& =\left[\left\langle\mu_{b_{i j}} \mu_{a_{i j}}\left(v_{b_{i j}}^{q}+v_{a_{i j}}^{q}-v_{b_{i j}}^{q} v_{a_{i j}}^{q}\right)^{1 / q}\right\rangle\right] \\
& =B \otimes_{q} A .
\end{aligned}
$$


(iii)

$$
\begin{aligned}
n\left(A \oplus_{q} B\right) & =n\left[\left\langle\left(\mu_{a_{i j}}^{q}+\mu_{b_{i j}}^{q}-\mu_{a_{i j}}^{q} \mu_{b_{i j}}^{q}\right)^{1 / q}, v_{a_{i j}} v_{b_{i j}}\right\rangle\right] \\
& =\left[\left\langle\left(1-\left[1-\left(\mu_{a_{i j}}^{q}+\mu_{b_{i j}}^{q}-\mu_{a_{i j}}^{q} \mu_{b_{i j}}^{q}\right)^{n}\right]^{1 / q},\left(v_{a_{i j}} v_{b_{i j}}\right)^{n}\right\rangle\right]\right. \\
& =\left[\left\langle\left(1-\left(1-\mu_{a_{i j}}^{q}\right)^{n}\left(1-\mu_{b_{i j}}^{q}\right)^{n}\right)^{1 / q},\left(v_{a_{i j}} v_{b_{i j}}\right)^{n}\right\rangle\right] \\
n A \oplus_{q} n B & =\left[\left\langle\left(1-\left(1-\mu_{a_{i j}}^{q}\right)^{n}\right)^{1 / q},\left(v_{a_{i j}}\right)^{n}\right\rangle \oplus_{q}\left\langle\left(1-\left(1-\mu_{a_{i j}}^{q}\right)^{n}\right)^{1 / q},\left(v_{a_{i j}}\right)^{n}\right\rangle\right] \\
& =\left[\left\langle\left(1-\left(1-\mu_{a_{i j}}^{q}\right)^{n}\left(1-\mu_{a_{i j}}^{q}\right)^{n}\right)^{1 / q},\left(v_{a_{i j}} v_{b_{i j}}\right)^{n}\right\rangle\right] \\
& =n\left(A \oplus_{q} B\right) .
\end{aligned}
$$

(iv)

$$
\begin{aligned}
\left(n_{1}+n_{2}\right) A & =\left[\left\langle\left(1-\left(1-\mu_{a_{i j}}^{q}\right)^{n_{1}+n_{2}}\right)^{1 / q},\left(v_{a_{i j}}\right)^{n_{1}+n_{2}}\right\rangle\right] \\
& =\left[\left\langle\left(1-\left(1-\mu_{a_{i j}}^{q}\right)^{n_{1}}\left(1-\mu_{a_{i j}}^{q}\right)^{n_{2}}\right)^{1 / q},\left(v_{a_{i j}} v_{b_{i j}}\right)^{n_{1}+n_{2}}\right\rangle\right] \\
& =\left[\left\langle\left(1-\left(1-\mu_{a_{i j}}^{q}\right)^{n_{1}}\right)^{1 / q},\left(v_{a_{i j}}\right)^{n_{1}}\right\rangle \oplus_{q}\left\langle\left(1-\left(1-\mu_{a_{i j}}^{q}\right)^{n_{2}}\right)^{1 / q},\left(v_{a_{i j}}\right)^{n_{2}}\right\rangle\right] \\
& =n_{1} A \oplus_{q} n_{2} A .
\end{aligned}
$$

(v)

$$
\begin{aligned}
\left(A \otimes_{q} B\right)^{n} & =\left[\left\langle\mu_{a_{i j}} \mu_{b_{i j}}\left(v_{a_{i j}}^{q}+v_{b_{i j}}^{q}-v_{a_{i j}}^{q} v_{b_{i j}}^{q}\right)^{1 / q}\right\rangle\right]^{n} \\
& =\left[\left\langle\left(\mu_{a_{i j}} \mu_{b_{i j}}\right)^{n},\left(1-\left(1-v_{a_{i j}}^{q}-v_{b_{i j}}^{q}+v_{a_{i j}}^{q} v_{b_{i j}}^{q}\right)^{n}\right)^{1 / q}\right\rangle\right]^{n} \\
& =\left[\left\langle\left(\mu_{a_{i j}}\right)^{n}\left(\mu_{b_{i j}}\right)^{n},\left(1-\left(1-\mu_{a_{i j}}^{q}\right)^{n}\left(1-\mu_{a_{i j}}^{q}\right)^{n}\right)^{1 / q}\right\rangle\right] \\
& =\left[\left\langle\mu_{a_{i j}}^{n}\left(1-\left(1-\mu_{a_{i j}}^{q}\right)^{n}\right)^{1 / q}\right\rangle \otimes_{q}\left\langle\mu_{b_{i j}}^{n}\left(1-\left(1-\mu_{b_{i j}}^{q}\right)^{n}\right)^{1 / q}\right\rangle\right] \\
& =A^{n} \otimes_{q} B^{n} .
\end{aligned}
$$

(vi)

$$
\begin{aligned}
A^{n_{1}} \otimes_{q} A^{n_{2}} & =\left[\left\langle\mu_{a_{i j^{\prime}}}^{n_{1}}\left(1-\left(1-\mu_{a_{i j}}^{q}\right)^{n_{1}}\right)^{1 / q}\right\rangle \otimes_{q}\left\langle\mu_{a_{i j^{\prime}}}^{n_{2}}\left(1-\left(1-\mu_{a_{i j}}^{q}\right)^{n_{2}}\right)^{1 / q}\right\rangle\right] \\
& =\left[\left\langle\mu_{a_{i j}}^{n_{1}+n_{2}},\left(1-\left(1-\mu_{a_{i j}}^{q}\right)^{n_{1}+n_{2}}\right)^{1 / q}\right\rangle\right] \\
& =A^{\left(n_{1}+n_{2}\right)} .
\end{aligned}
$$

Theorem 3. For $A, B \in Q_{m \times n}$, we have

(i) $A \wedge_{q} B=B \wedge_{q} A$,

(ii) $A \vee_{q} B=B \vee_{q} A$,

(iii) $A \wedge_{q}\left(B \wedge_{q} C\right)=\left(A \wedge_{q} B\right) \wedge_{q} C$,

(iv) $A \vee_{q}\left(B \vee_{q} C\right)=\left(A \vee_{q} B\right) \vee_{q} C$,

(v) $n\left(A \wedge_{q} B\right)=n A \wedge_{q} n B$,

(vi) $n\left(A \vee_{q} B\right)=n A \vee_{q} n B$,

(vii) $\left(A \wedge_{q} B\right)^{n}=A^{n} \wedge_{q} B^{n}$,

(viii) $\left(A \vee_{q} B\right)^{n}=A^{n} \vee_{q} B^{n}$.

Proof. Here we prove (i), (iii) and (vi). The remaining are similar. 
(i)

$$
\begin{aligned}
\left(A \wedge_{q} B\right) & =\left(\min \left\{\mu_{a_{i j}}, \mu_{b_{i j}}\right\}, \max \left\{v_{a_{i j}}, v_{b_{i j}}\right\}\right) \\
& =\left(\min \left\{\mu_{b_{i j}}, \mu_{a_{i j}}\right\}, \max \left\{v_{b_{i j}}, v_{a_{i j}}\right\}\right) \\
& =B \wedge_{q} A .
\end{aligned}
$$

(iii)

$$
\begin{aligned}
A \wedge_{q}\left(B \wedge_{q} C\right) & =\left(\mu_{a_{i j}}, v_{b_{i j}}\right) \wedge_{q}\left(\min \left\{\mu_{b_{i j}}, \mu_{c_{i j}}\right\}, \max \left\{v_{b_{i j}}, v_{c_{i j}}\right\}\right) \\
& =\left(\min \left\{\mu_{a_{i j}}, \min \left\{\mu_{b_{i j}}, \mu_{c_{i j}}\right\}\right\}, \max \left\{v_{a_{i j}}, \max \left\{v_{b_{i j}}, v_{c_{i j}}\right\}\right\}\right) \\
& =\left(\min \left\{\min \left\{\mu_{a_{i j}}, \mu_{b_{i j}}\right\}, \mu_{c_{i j}}\right\}, \max \left\{\max \left\{v_{a_{i j}}, v_{b_{i j}}\right\}, v_{c_{i j}}\right\}\right) \\
& =\left(\min \left\{\mu_{a_{i j}}, \mu_{b_{i j}}\right\}, \max \left\{v_{a_{i j}}, v_{b_{i j}}\right\}\right) \wedge_{q}\left(\mu_{c_{i j}}, v_{c_{i j}}\right) \\
& =\left(A \wedge_{q} B\right) \wedge_{q} C .
\end{aligned}
$$

(vi)

$$
\begin{aligned}
n\left(A \vee_{q} B\right) & =n A \vee_{q} n B \\
& =n\left(\min \left\{\mu_{a_{i j}}, \mu_{b_{i j}}\right\}, \max \left\{v_{a_{i j}}, v_{b_{i j}}\right\}\right) \\
& =\left[\left\langle\left(1-\left(1-\max \left\{\mu_{a_{i j}}^{q}, \mu_{b_{i j}}^{q}\right\}\right)^{n}\right)^{1 / q}, \min \left\{\left(v_{a_{i j}}\right)^{n},\left(v_{b_{i j}}\right)^{n}\right\}\right\rangle\right] \\
n A \vee_{q} n B & =\left[\left\langle\left(\left(1-\left(1-\mu_{a_{i j}}^{q}\right)^{n}\right)^{1 / q}, v_{a_{i j}}{ }^{n}\right) \vee\left(\left(1-\left(1-\mu_{a_{i j}}^{q}\right)^{n}\right)^{1 / q},\left(v_{a_{i j}}\right)^{n}\right)\right\rangle\right] \\
& =\left[\left\langle\max \left\{\left(1-\left(1-\mu_{a_{i j}}^{q}\right)^{n}\right)^{1 / q},\left(1-\left(1-\mu_{b_{i j}}^{q}\right)^{n}\right)^{1 / q}\right\}, \min \left\{\left(v_{a_{i j}}\right)^{n},\left(v_{b_{i j}}\right)^{n}\right\}\right\rangle\right] \\
& =\left[\left\langle\left(1-\left(1-\max \left\{\mu_{a_{i j}}^{q}, \mu_{b_{i j}}^{q}\right\}\right)^{n}\right)^{1 / q}, \min \left\{\left(v_{a_{i j}}\right)^{n},\left(v_{b_{i j}}\right)^{n}\right\}\right\rangle\right] \\
& =n\left(A \vee_{q} B\right) .
\end{aligned}
$$

Theorem 4. For $A, B \in Q_{m \times n}$, we have

(i) $\left(A \wedge_{q} B\right)^{C}=A^{C} \vee_{q} B^{C}$,

(ii) $\left(A \vee_{q} B\right)^{C}=A^{C} \wedge_{q} B^{C}$,

(iii) $\left(A \oplus_{q} B\right)^{C}=A^{C} \otimes_{q} B^{C}$,

(iv) $\left(A \otimes_{q} B\right)^{C}=A^{C} \oplus_{q} B^{C}$,

(v) $\left(A^{C}\right)^{n}=(n A)^{C}$,

(vi) $n\left(A^{C}\right)=\left(A^{n}\right)^{C^{\prime}}$.

Proof. Here we prove (i), (iii) and (iv). The remaining are similar.

(i)

$$
\begin{aligned}
\left(A \wedge_{q} B\right)^{C} & =\left[\left\langle\left(\min \left\{\mu_{a_{i j}}, \mu_{b_{i j}}\right\}, \max \left\{v_{a_{i j}}, v_{b_{i j}}\right\}\right)^{C}\right\rangle\right] \\
& =\left[\left\langle\max \left\{v_{a_{i j}}, v_{b_{i j}}\right\}, \min \left\{\mu_{a_{i j}}, \mu_{b_{i j}}\right\}\right\rangle\right] \\
& =\left(v_{a_{i j}}, \mu_{a_{i j}}\right) \vee_{q}\left(v_{b_{i j}}, \mu_{b_{i j}}\right) \\
& =A^{C} \vee_{q} B^{C} .
\end{aligned}
$$


(iii)

$$
\begin{aligned}
\left(A \oplus_{q} B\right)^{C} & =\left[\left\langle\left(\left(\mu_{a_{i j}}^{q}+\mu_{b_{i j}}^{q}-\mu_{a_{i j}}^{q} \mu_{b_{i j}}^{q}\right)^{1 / q}, v_{a_{i j}} v_{b_{i j}}\right)^{C}\right\rangle\right] \\
& =\left[\left\langle\mu_{a_{i j}} \mu_{b_{i j}}\left(v_{a_{i j}}^{q}+v_{b_{i j}}^{q}-v_{a_{i j}}^{q} v_{b_{i j}}^{q}\right)^{1 / q}\right\rangle\right] \\
& =\left(v_{a_{i j}} \mu_{b_{i j}}\right) \otimes\left(v_{b_{i j}} \mu_{b_{i j}}\right) \\
& =A^{C} \otimes_{q} B^{C} .
\end{aligned}
$$

(v)

$$
\begin{aligned}
\left(A^{C}\right)^{n} & =\left(v_{a_{i j}}, \mu_{a_{i j}}\right)^{n} \\
& =\left[\left\langle v_{a_{i j^{\prime}}}^{n}\left(1-\left(1-\mu_{a_{i j}}^{q}\right)^{n}\right)^{1 / q}\right\rangle\right] \\
& =\left[\left\langle\left(\left(1-\left(1-\mu_{a_{i j}}^{q}\right)^{n}\right)^{1 / q}, v_{a_{i j}}^{n}\right)^{C}\right\rangle\right] \\
& =(n A)^{C} .
\end{aligned}
$$

Theorem 5. For $A, B, C \in Q_{m \times n}$, we have
(i) $\left(A \vee_{q} B\right) \wedge_{q} C=\left(A \wedge_{q} C\right) \vee_{q}\left(B \wedge_{q} C\right)$,
(ii) $\left(A \wedge_{q} B\right) \vee_{q} C=\left(A \vee_{q} C\right) \wedge_{q}\left(B \vee_{q} C\right)$,
(iii) $\left(A \vee_{q} B\right) \oplus_{q} C=\left(A \oplus_{q} C\right) \vee_{q}\left(B \oplus_{q} C\right)$,
(iv) $\left(A \wedge_{q} B\right) \oplus_{q} C=\left(A \oplus_{q} C\right) \wedge_{q}\left(B \oplus_{q} C\right)$,
(v) $\left(A \vee_{q} B\right) \otimes_{q} C=\left(A \otimes_{q} C\right) \vee_{q}\left(B \otimes_{q} C\right)$,
(vi) $\left(A \wedge_{q} B\right) \otimes_{q} C=\left(A \otimes_{q} C\right) \wedge_{q}\left(B \otimes_{q} C\right)$.

Proof. Here we prove(i), (iii) and (v). The remaining can be proved analogously.

(i)

$$
\begin{aligned}
\left(A \vee_{q} B\right) \wedge_{q} C & =\left[\left\langle\min \left\{\max \left\{\mu_{a_{i j}}, \mu_{b_{i j}}\right\}, \mu_{c_{i j}}\right\}, \max \left\{\min \left\{v_{a_{i j}}, v_{b_{i j}}\right\} v_{c_{i j}}\right\}\right\rangle\right] \\
& =\left[\left\langle\max \left\{\min \left\{\mu_{a_{i j}}, \mu_{b_{i j}}\right\}, \min \left\{\mu_{a_{i j}}, \mu_{c_{i j}}\right\}\right\}, \min \left\{\max \left\{v_{a_{i j}}, v_{b_{i j}}\right\}, \max \left\{v_{b_{i j}}, v_{c_{i j}}\right\}\right\}\right\rangle\right] \\
& =\left[\left\langle\left\{\min \left\{\mu_{a_{i j}}, \mu_{c_{i j}}\right\}, \max \left\{v_{a_{i j}}, v_{c_{i j}}\right\}\right\} \vee\left\{\min \left\{v_{b_{i j}}, v_{c_{i j}}\right\}, \max \left\{v_{b_{i j}}, v_{c_{i j}}\right\}\right\}\right\rangle\right] \\
& =\left(A \wedge_{q} C\right) \vee_{q}\left(B \wedge_{q} C\right) .
\end{aligned}
$$

Hence, $\left(A \vee_{q} B\right) \wedge_{q} C=\left(A \wedge_{q} C\right) \vee_{q}\left(B \wedge_{q} C\right)$.

(iii)

$$
\begin{aligned}
\left(A \vee_{q} B\right) \oplus_{q} C & =\left(\max \left\{\mu_{a_{i j}}, \mu_{b_{i j}}\right\}, \min \left\{v_{a_{i j}}, v_{b_{i j}}\right\}\right) \oplus\left(\mu_{c_{i j}}, v_{c_{i j}}\right) \\
& =\left[\left\langle\left(\max \left\{\mu_{a_{i j}}^{q}, \mu_{b_{i j}}^{q}\right\}+\mu_{c_{i j}}^{q}-\max \left\{\mu_{a_{i j}}^{q}, \mu_{b_{i j}}^{q}\right\} \mu_{c_{i j}}^{q}\right)^{1 / q}, \min \left\{v_{a_{i j}}, v_{b_{i j}}\right\} v_{c_{i j}}\right\rangle\right] \\
& =\left[\left\langle\left(\left(1-\mu_{c_{i j}}^{q}\right) \max \left\{\mu_{a_{i j}}^{q}, \mu_{b_{i j}}^{q}\right\}+\mu_{c_{i j}}^{q}\right)^{1 / q}, \min \left\{v_{a_{i j}} v_{c_{i j}}, v_{b_{i j}} v_{c_{i j}}\right\}\right\rangle\right] . \\
\left(A \oplus_{q} C\right) \vee_{q}\left(B \oplus_{q} C\right) & =\left[\left\langle\max \left\{\left(\mu_{a_{i j}}^{q}+\mu_{c_{i j}}^{q}-\mu_{a_{i j}}^{q} \mu_{c_{i j}}^{q}\right)^{1 / q},\left(\mu_{b_{i j}}^{q}+\mu_{c_{i j}}^{q}-\mu_{b_{i j}}^{q} \mu_{c_{i j}}^{q}\right)^{1 / q}\right\}, \min \left\{v_{a_{i j}} v_{c_{i j}}, v_{b_{i j}} v_{c_{i j}}\right\}\right\rangle\right] \\
& =\left[\left\langle\max \left\{\left(\left(1-\mu_{c_{i j}}^{q}\right) \mu_{a_{i j}}^{q}+\mu_{c_{i j}}^{q}\right)^{1 / q},\left(\left(1-\mu_{c_{i j}}^{q}\right) \mu_{b_{i j}}^{q}+\mu_{c_{i j}}^{q}\right)^{1 / q}\right\}, \min \left\{v_{a_{i j}} v_{c_{i j}}, v_{b_{i j}} v_{c_{i j}}\right\}\right\rangle\right] \\
& =\left[\left\langle\left(\left(1-\mu_{c_{i j}}^{q}\right) \max \left\{\mu_{a_{i j}}^{q}, \mu_{b_{i j}}^{q}\right\}+\mu_{c_{i j}}^{q}\right)^{1 / q}, \min \left\{v_{a_{i j}} v_{c_{i j}}, v_{b_{i j}} v_{c_{i j}}\right\}\right\rangle\right] \\
& =\left(A \vee_{q} B\right) \oplus_{q} C .
\end{aligned}
$$


Hence, $\left(A \vee_{q} B\right) \oplus_{q} C=\left(A \oplus_{q} C\right) \vee_{q}\left(B \oplus_{q} C\right)$.

(v)

$$
\begin{aligned}
\left(A \vee_{q} B\right) \otimes_{q} C & =\left[\left\langle\max \left\{\mu_{a_{i j}}, \mu_{b_{i j}}\right\} \mu_{c_{i j}}\left(\min \left\{v_{a_{i j}}^{q}, v_{b_{i j}}^{q}\right\}+v_{c_{i j}}^{q}-\min \left\{v_{a_{i j}}^{q}, v_{b_{i j}}^{q}\right\} v_{c_{i j}}^{q}\right)^{1 / q}\right\rangle\right] \\
& =\left[\left\langle\max \left\{\mu_{a_{i j}}, \mu_{b_{i j}}\right\} \mu_{c_{i j}}\left(\left(1-v_{c_{i j}}^{q}\right) \min \left\{v_{a_{i j}}^{q}, v_{b_{i j}}^{q}\right\}+v_{c_{i j}}^{q}\right)^{1 / q}\right\rangle\right] . \\
\left(A \otimes_{q} C\right) \vee_{q}\left(B \otimes_{q} C\right) & =\left[\left\langle\max \left\{\mu_{a_{i j}} \mu_{c_{i j}}, \mu_{b_{i j}} \mu_{c_{i j}}\right\}, \min \left\{\left(v_{a_{i j}}^{q}+v_{c_{i j}}^{q}-v_{a_{i j}}^{q} v_{c_{i j}}^{q}\right)^{1 / q},\left(v_{b_{i j}}^{q}+v_{c_{i j}}^{q}-v_{b_{i j}}^{q} v_{c_{i j}}^{q}\right)^{1 / q}\right\}\right\rangle\right] \\
& =\left[\left\langle\max \left\{\mu_{a_{i j}} \mu_{c_{i j}}, \mu_{b_{i j}} \mu_{c_{i j}}\right\}, \min \left\{\left(\left(1-v_{c_{i j}}^{q}\right) v_{a_{i j}}^{q}+v_{c_{i j}}^{q}\right)^{1 / q},\left(\left(1-v_{c_{i j}}^{q}\right) v_{b_{i j}}^{q}+v_{c_{i j}}^{q}\right)^{1 / q}\right\}\right\rangle\right] \\
& =\left[\left\langle\max \left\{\mu_{a_{i j},} \mu_{b_{i j}}\right\} \mu_{c_{i j}}\left(\left(1-v_{c_{i j}}^{q}\right) \min \left\{v_{a_{i j}}^{q}, v_{b_{i j}}^{q}\right\}+v_{c_{i j}}^{q}\right)^{1 / q}\right\rangle\right] \\
& =\left(A \vee_{q} B\right) \otimes_{q} C .
\end{aligned}
$$

Hence, $\left(A \vee_{q} B\right) \otimes_{q} C=\left(A \otimes_{q} C\right) \vee_{q}\left(B \otimes_{q} C\right)$.

Theorem 6. For any q-ROFM A, we have

(i) $\left(A \oplus_{q} O\right)=\left(O \oplus_{q} A\right)=A$ ，

(ii) $\left(A \otimes_{q} J\right)=\left(J \otimes_{q} A\right)=A$.

Proof. (i) $A \oplus_{q} O=\left\langle\mu_{a_{i j}}, v_{a_{i j}}\right\rangle \oplus_{q}\langle 0,1\rangle=\left[\left\langle\left(\mu_{a_{i j}}^{q}+0-\mu_{a_{i j}}^{q} .0\right)^{1 / q}, v_{a_{i j}} .1\right\rangle\right]=\left[\left\langle\mu_{a_{i j}}, v_{a_{i j}}\right\rangle\right]=A$.

Similarly, we can prove $O \oplus_{q} A=A$.

(ii) $A \otimes_{q} J=\left\langle\mu_{a_{i j}}, v_{a_{i j}}\right\rangle \otimes_{q}\langle 1,0\rangle=\left[\left\langle\mu_{a_{i j} .1}\left(v_{a_{i j}}{ }^{q}+0-v_{a_{i j}}{ }^{q} .0\right)^{1 / q}\right\rangle\right]=\left[\left\langle\mu_{a_{i j}}, v_{a_{i j}}\right\rangle\right]=A$.

Similarly, we can prove $J \otimes_{q} A=A$.

Theorem 7. For any q-ROFM A, we have

(i) $\left(A \oplus_{q} J\right)=\left(J \oplus_{q} A\right)=J$,

(ii) $\left(A \otimes_{q} O\right)=\left(O \otimes_{q} A\right)=O$.

Proof. (i) $\left(A \oplus_{q} J\right)=\left\langle\mu_{a_{i j}}, v_{a_{i j}}\right\rangle \oplus_{q}\langle 1,0\rangle=\left[\left\langle\left(\mu_{a_{i j}}^{q}+1-\mu_{a_{i j}}^{q} \cdot 1\right)^{1 / q}, a_{i j} .0\right\rangle\right]=\langle 1,0\rangle=J$.

Similarly, we can prove $J \oplus_{q} A=J$.

(ii) $\left(A \otimes_{q} O\right)=\left\langle\mu_{a_{i j}}, v_{a_{i j}}\right\rangle \otimes_{q}\langle 0,1\rangle=\left[\left\langle\mu_{a_{i j}} .0,\left(v_{a_{i j}}^{q}+1-v_{a_{i j}}^{q} \cdot 1\right)^{1 / q}\right\rangle\right]=\langle 0,1\rangle=O$.

Similarly, we can prove $O \otimes_{q} A=O$.

\section{Necessity and Possibility operators on q-ROFMs}

In this section, we define necessity and possibility operators for q-ROFMs and proved their algebraic properties.

Definition 6. For every q-ROFM $A$, the necessity $(\square)$ and possibility $(\diamond)$ operators are defined as follows:

$$
\begin{aligned}
& \square A=\left[\left\langle\mu_{a_{i j},}\left(1-\mu_{a_{i j}}^{q}\right)^{1 / q}\right\rangle\right], \\
& \diamond A=\left[\left\langle\left(1-v_{a_{i j}}^{q}\right)^{1 / q}, v_{a_{i j}}\right\rangle\right] .
\end{aligned}
$$

Theorem 8. For $A, B \in Q_{m \times n}$, we have 
(i) $\square\left(A \oplus_{q} B\right)=\square A \oplus_{q} \square B$,

(ii) $\diamond\left(A \oplus_{q} B\right)=\diamond A \oplus_{q} \diamond B$.

Proof. (i)

$$
\begin{aligned}
\square\left(A \oplus_{q} B\right) & =\left[\left\langle\left(\mu_{a_{i j}}^{q}+\mu_{b_{i j}}^{q}-\mu_{a_{i j}}^{q} \mu_{b_{i j}}^{q}\right)^{1 / q},\left(1-\left(\mu_{a_{i j}}^{q}+\mu_{b_{i j}}^{q}-\mu_{a_{i j}}^{q} \mu_{b_{i j}}^{q}\right)\right)^{1 / q}\right\rangle\right] \\
\square A \oplus_{q} \square B & =\left[\left\langle\left(\mu_{a_{i j}}^{q}+\mu_{b_{i j}}^{q}-\mu_{a_{i j}}^{q} \mu_{b_{i j}}^{q}\right)^{1 / q},\left(1-\mu_{a_{i j}}^{q}\right)^{1 / q}\left(1-\mu_{b_{i j}}^{q}\right)^{1 / q}\right\rangle\right] \\
& =\left[\left\langle\left(\mu_{a_{i j}}^{q}+\mu_{b_{i j}}^{q}-\mu_{a_{i j}}^{q} \mu_{b_{i j}}^{q}\right)^{1 / q},\left(1-\left(\mu_{a_{i j}}^{q}+\mu_{b_{i j}}^{q}-\mu_{a_{i j}}^{q} \mu_{b_{i j}}^{q}\right)\right)^{1 / q}\right\rangle\right] .
\end{aligned}
$$

Hence, $\square\left(A \oplus_{q} B\right)=\square A \oplus_{q} \square B$.

(ii)

$$
\begin{aligned}
& \diamond\left(A \oplus_{q} B\right)=\left[\left\langle\left(1-v_{a_{i j}}^{q} v_{b_{i j}}^{q}\right)^{1 / q}, v_{a_{i j}} v_{b_{i j}}\right\rangle\right] \\
& \diamond A \oplus_{q} \diamond B=\left[\left\langle\left(\left(1-v_{a_{i j}}^{q}\right)+\left(1-v_{b_{i j}}^{q}\right)-\left(1-v_{a_{i j}}^{q}\right)\left(1-v_{b_{i j}}^{q}\right)\right)^{1 / q}, v_{a_{i j}} v_{b_{i j}}\right\rangle\right]=\left[\left\langle\left(1-v_{a_{i j}}^{q} v_{b_{i j}}^{q}\right)^{1 / q}, v_{a_{i j}} v_{b_{i j}}\right\rangle\right] .
\end{aligned}
$$

Hence, $\diamond\left(A \oplus_{q} B\right)=\diamond A \oplus_{q} \diamond B$.

Theorem 9. For $A, B \in Q_{m \times n}$, we have

(i) $\square\left(A \otimes_{q} B\right)=\square A \otimes_{q} \square B$,

(ii) $\diamond\left(A \otimes_{q} B\right)=\diamond A \otimes_{q} \diamond B$.

Proof. (i)

$$
\begin{aligned}
\square\left(A \otimes_{q} B\right) & =\left[\left\langle\mu_{a_{i j}} \mu_{b_{i j}}\left(1-\mu_{a_{i j}}^{q} \mu_{b_{i j}}^{q}\right)^{1 / q}\right\rangle\right] \\
\square A \otimes_{q} \square B & =\left[\left\langle\mu_{a_{i j}} \mu_{b_{i j}}\left(\left(1-\mu_{a_{i j}}^{q}\right)+\left(1-\mu_{b_{i j}}^{q}\right)-\left(1-\mu_{a_{i j}}^{q}\right)\left(1-\mu_{b_{i j}}^{q}\right)\right)^{1 / q}\right\rangle\right] \\
& =\left[\left\langle\mu_{a_{i j}} \mu_{b_{i j}}\left(1-\mu_{a_{i j}}^{q} \mu_{b_{i j}}^{q}\right)^{1 / q}\right\rangle\right] .
\end{aligned}
$$

Hence, $\square\left(A \otimes_{q} B\right)=\square A \otimes_{q} \square B$.

(ii) It can be proved analogously.

Theorem 10. For $A, B \in Q_{m \times n}$, we have

(i) $\left(\square\left(A^{C} \oplus_{q} B^{C}\right)\right)^{C}=\diamond A \otimes_{q} \diamond B$,

(ii) $\left(\square\left(A^{C} \otimes_{q} B^{C}\right)\right)^{C}=\diamond A \oplus_{q} \diamond B$.

Proof. (i)

$$
\begin{gathered}
\left(A^{C} \oplus_{q} B^{C}\right)=\left[\left\langle\left(v_{a_{i j}}^{q}+v_{b_{i j}}^{q}-v_{a_{i j}}^{q} v_{b_{i j}}^{q}\right)^{1 / q}, \mu_{a_{i j}} \mu_{b_{i j}}\right\rangle\right], \\
\square\left(A^{C} \oplus_{q} B^{C}\right)=\left[\left\langle\left(v_{a_{i j}}^{q}+v_{b_{i j}}^{q}-v_{a_{i j}}^{q} v_{b_{i j}}^{q}\right)^{1 / q},\left(1-\left(v_{a_{i j}}^{q}+v_{b_{i j}}^{q}-v_{a_{i j}}^{q} v_{b_{i j}}^{q}\right)\right)^{1 / q}\right\rangle\right], \\
\left(\square\left(A^{C} \oplus_{q} B^{C}\right)\right)^{C}=\left[\left\langle\left(1-\left(v_{a_{i j}}^{q}+v_{b_{i j}}^{q}-v_{a_{i j}}^{q} v_{b_{i j}}^{q}\right)\right)^{1 / q},\left(v_{a_{i j}}^{q}+v_{b_{i j}}^{q}-v_{a_{i j}}^{q} v_{b_{i j}}^{q}\right)^{1 / q}\right\rangle\right]=\diamond A \otimes_{q} \diamond B .
\end{gathered}
$$

(ii) It can be proved analogously.

Theorem 11. For $A, B \in Q_{m \times n}$, we have 
(i) $\left(\diamond\left(A^{C} \oplus_{q} B^{C}\right)\right)^{C}=\square A \otimes_{q} \square B$,

(ii) $\left(\diamond\left(A^{C} \otimes_{q} B^{C}\right)\right)^{C}=\square A \oplus_{q} \square B$.

Proof. (i)

$$
\begin{aligned}
& \diamond\left(A^{C} \oplus_{q} B^{C}\right)=\left[\left\langle\left(1-\mu_{a_{i j}}^{q} \mu_{b_{i j}}^{q}\right)^{1 / q}, \mu_{a_{i j}} \mu_{b_{i j}}\right\rangle\right], \\
&\left(\diamond\left(A^{C} \oplus_{q} B^{C}\right)\right)^{C}=\left[\left\langle\mu_{a_{i j}} \mu_{b_{i j}}\left(1-\mu_{a_{i j}}^{q} \mu_{b_{i j}}^{q}\right)^{1 / q}\right\rangle\right]=\square A \otimes_{q} \square B .
\end{aligned}
$$

(ii) It can be proved similarly.

\section{New operation (@) on q-ROFMs}

In this section, we define the @ operation on q-ROFMs and present their algebraic properties. We discuss the Distributivity law in the case the operation of Algebraic sum and Algebraic product, $\vee_{q}$ and $\wedge_{q}$ are combined each other.

Definition 7. Let $A=\left[\left\langle\mu_{a_{i j}}, v_{a_{i j}}\right\rangle\right]$, and $B=\left[\left\langle\mu_{i_{i j}}, v_{b_{i j}}\right\rangle\right]$ be any two q-ROFMs. The new operation of q-ROFM is defined by

$$
A @ B=\left[\left\langle\left(\frac{\mu_{a_{i j}}^{q}+\mu_{b_{i j}}^{q}}{2}\right)^{1 / q},\left(\frac{v_{a_{i j}}^{q}+v_{b_{i j}}^{q}}{2}\right)^{1 / q}\right\rangle\right] .
$$

Theorem 12. For any $q$-ROFM $A$, we have $A @ A=A$.

\section{Proof.}

$$
\begin{aligned}
A @ A & =\left[\left\langle\left(\frac{\mu_{a_{i j}}^{q}+\mu_{a_{i j}}^{q}}{2}\right)^{1 / q},\left(\frac{v_{a_{i j}}^{q}+v_{a_{i j}}^{q}}{2}\right)^{1 / q}\right\rangle\right] \\
& =\left[\left\langle\left(\frac{2 \mu_{a_{i j}}^{q}}{2}\right)^{1 / q},\left(\frac{2 v_{a_{i j}}^{q}}{2}\right)^{1 / q}\right\rangle\right] \\
& =\left[\left\langle\mu_{a_{i j}}^{q}, v_{a_{i j}}^{q}\right\rangle\right] \\
& =\left[\left\langle\mu_{a_{i j}}, v_{a_{i j}}\right\rangle\right] \\
& =A .
\end{aligned}
$$

Theorem 13. For $A, B \in Q_{m \times n}$, we have
(i) $\left(A \oplus_{q} B\right) \wedge_{q}\left(A \otimes_{q} B\right)=A \otimes_{q} B$ ，
(ii) $\left(A \oplus_{q} B\right) \vee_{q}\left(A \otimes_{q} B\right)=A \oplus_{q} B$,
(iii) $\left(A \oplus_{q} B\right) \wedge_{q}(A @ B)=A @ B$,
(iv) $\left(A \oplus_{q} B\right) \vee_{q}(A @ B)=A \oplus_{q} B$,
(v) $\left(A \otimes_{q} B\right) \wedge_{q}(A @ B)=A \otimes_{q} B$,
(vi) $\left(A \otimes_{q} B\right) \vee_{q}(A @ B)=A @ B$.

Proof. (i)

$$
\begin{aligned}
\left(A \oplus_{P} B\right) \wedge_{q}\left(A \otimes_{q} B\right) & =\left[\left\langle\min \left\{\left(\mu_{a_{i j}}^{q}+\mu_{b_{i j}}^{q}-\mu_{a_{i j}}^{q} \mu_{b_{i j}}^{q}\right)^{1 / q}, \mu_{a_{i j}} \mu_{b_{i j}}\right\}, \max \left\{v_{a_{i j}} v_{b_{i j}}\left(v_{a_{i j}}^{q}+v_{b_{i j}}^{q}-v_{a_{i j}}^{q} v_{b_{i j}}^{q}\right)^{1 / q}\right\}\right\rangle\right] \\
& =\left[\left\langle\mu_{a_{i j}} \mu_{b_{i j}}\left(v_{a_{i j}}^{q}+v_{b_{i j}}^{q}-v_{a_{i j}}^{q} v_{b_{i j}}^{q}\right)^{1 / q}\right\rangle\right] \\
& =A \otimes_{q} B .
\end{aligned}
$$


Hence, $\left(A \oplus_{q} B\right) \wedge_{q}\left(A \otimes_{q} B\right)=A \otimes_{q} B$.

(ii)

$$
\begin{aligned}
\left(A \oplus_{q} B\right) \vee_{q}\left(A \otimes_{q} B\right) & =\left[\left\langle\max \left\{\left(\mu_{a_{i j}}^{q}+\mu_{b_{i j}}^{q}-\mu_{a_{i j}}^{q} \mu_{b_{i j}}^{q}\right)^{1 / q}, \mu_{a_{i j}} \mu_{b_{i j}}\right\}, \min \left\{v_{a_{i j}} v_{b_{i j}}\left(v_{a_{i j}}^{q}+v_{b_{i j}}^{q}-v_{a_{i j}}^{q} v_{b_{i j}}^{q}\right)^{1 / q}\right\}\right\rangle\right] \\
& =\left[\left\langle\left(\mu_{a_{i j}}^{q}+\mu_{b_{i j}}^{q}-\mu_{a_{i j}}^{q} \mu_{b_{i j}}^{q}\right)^{1 / q}, v_{a_{i j}} v_{b_{i j}}\right\rangle\right] \\
& =A \oplus_{q} B .
\end{aligned}
$$

Hence, $\left(A \oplus_{q} B\right) \vee_{q}\left(A \otimes_{q} B\right)=A \oplus_{q} B$.

(iii)

$$
\begin{aligned}
\left(A \oplus_{q} B\right) \wedge_{q}(A @ B) & =\left[\left\langle\min \left\{\left(\mu_{a_{i j}}^{q}+\mu_{b_{i j}}^{q}-\mu_{a_{i j}}^{q} \mu_{b_{i j}}^{q}\right)^{1 / q},\left(\frac{\mu_{a_{i j}}^{q}+\mu_{b_{i j}}^{q}}{2}\right)^{1 / q}\right\}, \max \left\{v_{a_{i j}} v_{b_{i j}}\left(\frac{v_{a_{i j}}^{q}+v_{b_{i j}}^{q}}{2}\right)^{1 / q}\right\}\right\rangle\right] \\
& =\left[\left\langle\left(\frac{\mu_{a_{i j}}^{q}+\mu_{b_{i j}}^{q}}{2}\right)^{1 / q},\left(\frac{v_{a_{i j}}^{q}+v_{b_{i j}}^{q}}{2}\right)^{1 / q}\right\rangle\right] \\
& =A @ B .
\end{aligned}
$$

Hence, $\left(A \oplus_{q} B\right) \wedge_{q}(A @ B)=A @ B$.

(iv)

$$
\begin{aligned}
\left(A \oplus_{q} B\right) \vee_{q}(A @ B) & =\left[\left\langle\max \left\{\left(\mu_{a_{i j}}^{q}+\mu_{b_{i j}}^{q}-\mu_{a_{i j}}^{q} \mu_{a_{i j}}^{q}\right)^{1 / q},\left(\frac{\mu_{a_{i j}}^{q}+\mu_{b_{i j}}^{q}}{2}\right)^{1 / q}\right\}, \min \left\{v_{a_{i j}} v_{b_{i j}}\left(\frac{v_{a_{i j}}^{q}+v_{b_{i j}}^{q}}{2}\right)^{1 / q}\right\}\right\rangle\right] \\
& =\left[\left\langle\left(\mu_{a_{i j}}^{q}+\mu_{b_{i j}}^{q}-\mu_{a_{i j}}^{q} \mu_{b_{i j}}^{q}\right)^{1 / q}, v_{a_{i j}} v_{b_{i j}}\right\rangle\right] \\
& =A \oplus_{q} B .
\end{aligned}
$$

Hence, $\left(A \oplus_{q} B\right) \vee_{q}(A @ B)=A \oplus_{q} B$.

(v)

$$
\begin{aligned}
\left(A \otimes_{q} B\right) \wedge_{q}(A @ B) & =\left[\left\langle\min \left\{\mu_{a_{i j}} \mu_{b_{i j}}\left(\frac{\mu_{a_{i j}}^{q}+\mu_{b_{i j}}^{q}}{2}\right)^{1 / q}\right\}, \max \left\{\left(v_{a_{i j}}^{q}+v_{b_{i j}}^{q}-v_{a_{i j}}^{q} v_{b_{i j}}^{q}\right)^{1 / q},\left(\frac{v_{a_{i j}}^{q}+v_{b_{i j}}^{q}}{2}\right)^{1 / q}\right\}\right\rangle\right] \\
& =\left[\left\langle\mu_{a_{i j}} \mu_{b_{i j}}\left(v_{a_{i j}}^{q}+v_{b_{i j}}^{q}-v_{a_{i j}}^{q} v_{b_{i j}}^{q}\right)^{1 / q}\right\rangle\right] \\
& =A \otimes_{q} B .
\end{aligned}
$$

Hence, $\left(A \otimes_{q} B\right) \wedge_{q}(A @ B)=A \otimes_{q} B$.

(vi)

$$
\begin{aligned}
\left(A \otimes_{q} B\right) \vee_{q}(A @ B) & =\left[\left\langle\max \left\{\mu_{a_{i j}} \mu_{b_{i j}}\left(\frac{\mu_{a_{i j}}^{q}+\mu_{b_{i j}}^{q}}{2}\right)^{1 / q}\right\}, \min \left\{\left(v_{a_{i j}}^{q}+v_{b_{i j}}^{q}-v_{a_{i j}}^{q} v_{b_{i j}}^{q}\right)^{1 / q},\left(\frac{v_{a_{i j}}^{q}+v_{b_{i j}}^{q}}{2}\right)^{1 / q}\right\}\right\rangle\right] \\
& =\left[\left\langle\left(\frac{\mu_{a_{i j}}^{q}+\mu_{b_{i j}}^{q}}{2}\right)^{1 / q},\left(\frac{v_{a_{i j}}^{q}+v_{b_{i j}}^{q}}{2}\right)^{1 / q}\right\rangle\right] \\
& =A @ B .
\end{aligned}
$$

Hence, $\left(A \otimes_{q} B\right) \vee_{q}(A @ B)=A @ B$. 
Remark 1. The q-rung orthopair fuzzy matrix forms a commutative monoid, associativity, commutativity and identity under the q-rung orthopair fuzzy matrix operation of algebraic sum and algebraic product. The distributive law also holds for $\oplus_{q}, \otimes_{q}$ and $\wedge_{q}, \vee_{q}$, are combined each other.

\section{Application}

The formation of $\mathrm{q}$-ROFMs is commutative monoid structure, q-rung orthopair fuzzy matrix and algebraic structure on this matrix, the results are applicable.

\section{Conclusion}

Generalized orthopair fuzzy matrices are extensions of intuitionistic fuzzy matrices and Pythagorean fuzzy matrices. Each element is expressed as an ordered pair of values, the former indicating the support for membership and the latter support against membership. The restriction on the memberships is that the sum of the $q^{\text {th }}$ powers of the support for and support against is equal to or less than one. Thus it greatly increases the modelers' ability to capture their judgment of the appropriate orthopair membership grade. In this paper, we proposed q-rung orthopair fuzzy matrices and its algebraic operations are defined. Then we proved some algebraic properties of $\mathrm{q}-\mathrm{ROFMs}$, such as associativity, commutativity, identity, distributivity and De Morgan's laws over complement. Furthermore, we defined necessity and possibility operators on q-ROFMs and investigated their algebraic properties. Finally, a new operation(@) on q-ROFMs are defined and discussed distributive laws in the case where the operations of $\oplus_{q}, \otimes_{q}, \wedge_{q}$ and $\vee_{q}$ are combined each other. For the development of q-rung orthopair fuzzy commutative monoid structure and its algebraic property the results of this paper would be helpful.

Conflicts of Interest: "The author declares no conflict of interest".

\section{References}

[1] Khan, S. K., Pal, M., \& Shyamal, A. K. (2002). Intuitionistic fuzzy matrices. Notes on Intuitionistic Fuzzy Sets, 8(2), 51-62.

[2] Im, Y. P., Lee, F. B., \& Park, S. W. (2001). The determinant of square intuitionistic fuzzy matrices. Far East Journal of Mathematical Science, 3(5), 789-796.

[3] Thomason, M. G. (1977). Convergence of powers of Fuzzy matrix. Journal of Mathematical Analysis and Applications, $57(2), 476-480$.

[4] Khan, S. K., \& Pal, M. (2006). Some operations on intuitionistic fuzzy matrices. Acta Ciencia Indica, 32, 515-524.

[5] Pal, P. (2001). Intuitionistic fuzzy determinant. V.U.J. Physical Sciences, 7, 87-93.

[6] Mondal, S., \& Pal, M. (2013). Similarity relations, invertibility and eigenvalues of IFM. Fuzzy Information and Engineering, 5(4), 431-443.

[7] Muthuraji, T., Sriram, S., \& Murugadas, P. (2016). Decomposition of intuitionistic fuzzy matrices. Fuzzy Information and Engineering, 8(3), 345-354.

[8] Sriram, S., \& Boobalan, J. (2016). Monoids of intuitionistic fuzzy matrices. Annals of Fuzzy Mathematics and Informatics, 11(3), 505-510.

[9] Muthuraji, T., \& Sriram, S. (2017). Representation and decomposition of an intuitionistic fuzzy matrix using some $\left(\alpha, \alpha^{\prime}\right)$ cuts. Applications and Applied Mathematics, 12(1), 241-258.

[10] Silambarasan, I., \& Sriram, S. (2017). Hamacher sum and Hamacher product of fuzzy matrices. International Journal of Fuzzy Mathematical Archive, 13(2), 191-198.

[11] Silambarasan, I., \& Sriram, S. (2018). Hamacher operations of intuitionistic fuzzy matrices. Annals of Pure and Applied Mathematics, 16(1), 81-90.

[12] Silambarasan, I. (2020). Interval-valued intuitionistic fuzzy matrices based on Hamacher operations. World Scientific News, 150, 148-161.

[13] Silambarasan, I. (2020). Some operations over interval-valued fuzzy matrices. Journal of Science, Computing and Engineering Research, 1(5), 131-137.

[14] Silambarasan, I., \& Sriram, S. (2021). Some operations over intuitionistic fuzzy matrices based on Hamacher t-norm and t-conorm. TWMS Journal of Applied and Engineering Mathematics, 11(2), 541-551.

[15] Yager, R. R. (2014). Pythagorean membership grades in multi-criteria decision making. IEEE Transactions on Fuzzy Systems, 22(4), 958-965.

[16] Zhang, X. L., \& Xu, Z. S. (2014). Extension of TOPSIS to multiple criteria decision making with Pythagorean fuzzy sets. International Journal of Intelligent Systems, 29(12), 1061-1078.

[17] Yager, R. R. (2017). Generalized orthopair fuzzy sets. IEEE Transactions on Fuzzy Systems, 25(5), 1222-1230. 
[18] Liu, P., \& Wang, P. (2018). Some q-rung orthopair fuzzy aggregation operators and their applications to multiple-attribute decision making. International Journal of Intelligent Systems, 33(2), 259-280.

[19] Riaz, M., Pamucar, D., Athar Farid, H. M., \& Hashmi, M. R. (2020). q-Rung orthopair fuzzy prioritized aggregation operators and their application towards green supplier chain management. Symmetry, 12, Article No. 976, https://doi.org/10.3390/sym12060976.

[20] Riaz, M., Athar Farid, H. M., Karaaslan, F., \& Hashmi, M. R. (2020). Some q-rung orthopair fuzzy hybrid aggregation operators and TOPSIS method for multi-attribute decision-making. Journal of Intelligent $\mathcal{E}$ Fuzzy Systems, 39(1), 1227-1241.

[21] Riaz, M., Athar Farid, H. M., Kalsoom, H., Pamucar, D., \& Chu, Y.M.(2020). A robust q-rung orthopair fuzzy Einstein prioritized aggregation operators with application towards MCGDM. Symmetry, 12, Article No. 1058, https://doi.org/10.3390/sym12061058.

[22] Riaz, M., Garg, H., Athar Farid, H. M., \& Chinram, R. (2021). Multi-criteria decision making based on bipolar picture fuzzy operators and new distance measures. CMES-Computer Modeling in Engineering E Sciences, 127(2), 771-800.

[23] Feng, F., Zheng, Y., \& Sun, B. et al.(2021). Novel score functions of generalized orthopair fuzzy membership grades with application to multiple attribute decision making. Granular Computing, https://doi.org/10.1007/s41066-021-00253-7.

[24] Akram, M., Alsulami, S., Karaaslan, F., \& Khan, A. (2021). q-Rung orthopair fuzzy graphs under Hamacher operators. Journal of Intelligent \& Fuzzy Systems, 40(1), 1367-1390.

[25] Akram, M., Shahzadi, G. \& Peng, X. (2020). Extension of Einstein geometric operators to multi-attribute decision making under q-rung orthopair fuzzy information. Granular Computing, https:/ /doi.org/10.1007/s41066-020-00233-3.

[26] Silambarasan, I., \& Sriram, S.(2018). Algebraic operations on Pythagorean fuzzy matrices. Mathematical Sciences International Research Journal, 7(2), 406-414.

[27] Silambarasan, I., \& Sriram, S.(2019). Commutative monoid of Pythagorean fuzzy matrices. International Journal of Computer Sciences and Engineering, 7(4), 637-642.

[28] Silambarasan, I., \& Sriram, S. (2019). New Operations for Pythagorean Fuzzy Matrices. Indian Journal of Science and Technology, 12(20), 1-7.

(C) 2021 by the authors; licensee PSRP, Lahore, Pakistan. This article is an open access article distributed under the terms and conditions of the Creative Commons Attribution (CC-BY) license (http://creativecommons.org/licenses/by/4.0/). 